\title{
Malignant pleural and pericardial effusions and meningeal infiltrates without other metastases in breast cancer: A case report
}

\author{
XINGXING LV ${ }^{1}$, JINLAN HE ${ }^{1}$, YUAN SHEN ${ }^{2}$ and HONG ZHENG ${ }^{1}$ \\ ${ }^{1}$ Department of Head \& Neck and Mammary Oncology and Medical Oncology, \\ Cancer Center and State Key Laboratory of Biotherapy, Laboratory of Molecular Diagnosis of Cancer, \\ West China Hospital; ${ }^{2}$ Department of General Practice, West China School of Clinical Medicine, \\ Sichuan University, Chengdu, Sichuan 610041, P.R. China
}

Received October 7, 2015; Accepted February 25, 2016

DOI: $10.3892 / \mathrm{mco} .2016 .798$

\begin{abstract}
Breast cancer metastasizing to the pleura, pericardium and leptomeninges, but not to other sites, is rare. Although malignant pericardial and pleural effusions are common complications during the course of malignancies, they are rarely the initial manifestations of malignant disease, particularly pericardial effusion with cardiac tamponade. This report describes a case of breast carcinoma in a 44-year-old woman who initially presented with malignant pleural effusion and pericardial tamponade and suffered from meningeal metastases after 4.5 months. Unfortunately, the patient succumbed to pericardial tamponade 7 months later. There was no metastasis identified in other organs during the course of the disease. To the best of our knowledge, no similar case has been reported in the literature to date.
\end{abstract}

\section{Introduction}

The most common sites of metastatic breast cancer are the brain, lungs, liver, soft tissue and bone (1). Although less frequent than solid organ and bone metastasis, malignant effusion, including pleural and pericardial effusion, and meningeal infiltrates are somewhat common in breast cancer. Approximately $7-11 \%$ of patients with breast cancer develop malignant pleural effusion during the course of the disease (2), and in $43 \%$ of these patients, pleural effusion is the first symptom of metastatic disease (3). The prevalence of pericardial involvement varies from $4 \%$ in general autopsies to $15-30 \%$ in autopsies of cancer patients (4). Only $12-25 \%$ of patients who have metastasis to the pericardium develop pericardial effusion, of whom only

Correspondence to: Professor Hong Zheng, Department of Head \& Neck and Mammary Oncology and Medical Oncology, Cancer Center and State Key Laboratory of Biotherapy, Laboratory of Molecular Diagnosis of Cancer, West China Hospital, Sichuan University, 37 Guo Xue Xiang, Chengdu, Sichuan 610041, P.R. China E-mail: hzheng@scu.edu.cn

Key words: breast cancer, malignant pleural effusion, malignant pericardial effusion, meningeal metastasis, pericardial tamponade a small percentage develop tamponade (5). Therefore, there are few reported cases of cardiac metastases from breast cancer. Pericardial effusion with cardiac tamponade as the initial manifestation of breast cancer is quite rare. The estimated incidence of leptomeningeal metastasis in clinical and autopsy series of patients with breast cancer is 2-5 and 3-6\%, respectively (6). The incidence of metastasis to the pleura, pericardium or meninges is lower compared with those to other sites in breast cancer, whereas simultaneous involvement of all three sites in one patient, without metastases to other sites, is extremely rare. To the best of our knowledge, no similar cases have been reported in the literature to date.

It has been reported that $\sim 3-6 \%$ of new breast cancer cases have already metastasized to distant sites at the time of diagnosis (7), with the median survival ranging between 18 and 39 months. Several studies have reported a range of prognostic factors affecting survival, such as age at diagnosis, hormone receptor status, human epidermal growth factor receptor 2 status and site of metastasis (8). Negative hormone receptor expression, cardiac tamponade and pleural effusion as the initial manifestation, and meningeal metastases, are poor prognostic factors.

\section{Case report}

A 44-year-old woman presented to the West China Hospital (Chengdu, China) in October, 2013 complaining of pain in the right breast for 2 months, a mass in the right breast, progressive dyspnea and productive cough accompanied by general malaise for 1 month. Prior to admission, a plain chest computed tomography $(\mathrm{CT})$ scan revealed bilateral pleural effusions and a large amount of pericardial effusion with characteristics of tamponade (Fig. 1). The cytological examination of the pleural and pericardial effusions demonstrated atypical cells suspicious of breast ductal carcinoma metastasis. Thoracentesis and pericardiocentesis were performed and drainage tubes were placed. The dyspnea and cough were significantly alleviated following drainage of the fluid and the subsequent intrathoracic and intraperitoneal administration of cisplatin. A color Doppler ultrasound revealed a firm mass $(33 \times 19 \times 32 \mathrm{~mm})$ in the right breast and several enlarged lymph nodes in the right axilla. The biopsy 
and subsequent histological examination of the mass revealed an infiltrative carcinoma (estrogen receptor, $0 \%$; progesterone receptor, 0\%; Ki-67, 20\%; and human epidermal growth factor receptor 2, 1+). Further examinations, including abdominal ultrasound, cranial CT scan and bone scan, detected no other metastasis. Upon admission, re-examination with color Doppler ultrasound revealed that the breast mass had increased to $52 \times 23 \times 46 \mathrm{~mm}$ in size over a few days. On the basis of these results, the patient was diagnosed with invasive ductal breast carcinoma (cT3N2M1) of the right breast, with malignant pleural and pericardial effusion.

The patient was then treated with docetaxel $\left(75 \mathrm{mg} / \mathrm{m}^{2}\right.$, day 1$)$ and gemcitabine $\left(1,000 \mathrm{mg} / \mathrm{m}^{2}\right.$, days 1 and 8$)$ every 3 weeks, for 2 cycles, achieving stable disease. Subsequently, the chemotherapy was changed to carboplatin (400 mg, day 1) and vinorelbine (40 mg/m $\mathrm{m}^{2}$, days 1 and 8 ) every 3 weeks, achieving partial response after the second cycle. Prior to receiving the third cycle, the patient complained of headache, dizziness and tinnitus, but no disorder of consciousness. An enhanced magnetic resonance imaging (MRI) scan of the head revealed absence of metastases; palliative therapy did not mitigate the symptoms. Several days later, the patient presented with exacerbation of the headache, diplopia, nausea, vomiting, convulsions, palpitations and dyspnea. On physical examination, the patient was dyspnoeic, with diminished breath sounds on the right pulmonary field and muffled cardiac tones. A chest-abdominal CT scan was performed, which revealed a large amount of pericardial effusion and a moderate amount of right pleural effusion (Fig. 2). Lumbar puncture was performed and revealed elevated opening pressure, protein, and lymphocytes, and reduced glucose, but without evidence of malignant cells in the cerebrospinal fluid (CSF). Thoracentesis and pericardiocentesis were performed and drainage tubes were again placed. In addition, treatments for reducing the intracranial pressure and antiepileptic agents were also administered. With these measures, the symptoms were controlled. However, symptoms of meningeal metastasis reappeared only 10 days later. The head MRI scan this time revealed narrowed bilateral frontal lobe grooves, noticeable hydrocephalus and enlarged ventricles; the enhanced scan revealed mild enhancement of the gyrus, suggesting meningeal metastasis (Fig. 3). Therefore, whole-brain radiotherapy (WBRT) with a dose of 40 Gy in 20 fractions was administered, with supportive treatment with mannitol, glycerin fructose, sodium valproate, levetiracetam and nutritional support. The patient's consciousness improved with these treatments. However, the patient's heart rate increased from 90-100 to 120-130 beats per minute, accompanied by dyspnea; re-examination with chest CT scan revealed a large amount of pericardial and pleural effusion (Fig. 4). Pericardial cavity drainage and intracavitary cisplatin was administered 3 times.

Unfortunately, the general condition of the patient deteriorated with rapid progression of the pericardial effusion and the patient succumbed to pericardial tamponade on May 3, 2014.

\section{Discussion}

It is well known that breast cancer spreads to different distant organs, preferentially to the bones, lungs, liver, soft tissues and brain, whereas metastasis to the pleura, pericardium or meninges is less common. Absence of metastasis to organs other than the pleura, pericardium and meninges during the entire course of the disease is extremely rare and, to the best of our knowledge, this is the first report of such a case. Of note, there was no solid evidence for meningeal metastases in the present report, as no neoplastic cells were found in the CSF examination and no obvious metastatic sites were identified on MRI. However, a negative MRI following negative CSF cytology cannot exclude meningeal metastases. It was previously reported that the first lumbar puncture exhibits low sensitivity, identifying neoplastic cells only in $45 \%$ of patients with meningeal metastases, whereas $\leq 40 \%$ of patients with clinically suspected meningeal metastases proven at the time of autopsy have negative CSF cytology; in addition, the false-negative rate of MRI is $30 \%$ (9). Thus, we may infer the presence of meningeal metastases in this patient based on indirect evidence: First, the patient displayed typical clinical characteristics, including headache, giddiness, tinnitus, diplopia, nausea, vomiting, convulsions and disorders of consciousness; second, although no neoplastic cells were found on CSF examination and no obvious metastatic sites were identified on MRI, there was elevated intracranial pressure, protein and lymphocytes, and decreased glucose on CSF examination, as well as noticeable hydrocephalus and enlarged ventricles on MRI. Finally, the patient's mental status improved and the clinical symptoms were alleviated following WBRT. The molecular basis of the metastatic process in breast cancer, particularly the mechanism underlying organ-specific metastasis, is poorly understood. Recent research data suggested that primary tumor gene signatures predict tumor metastatic potential and organ-specific tropism (10). The mechanism underlying breast cancer metastasis in this patient may be different from other common types of cancer, and it requires extensive investigation. However, the patient's family members refused the autopsy; thus, no further specimens were obtained to investigate the potential mechanisms.

Although metastatic breast cancer remains an incurable disease, survival has improved in recent years, due to a wide range of new therapeutic agents and improved supportive care. Although rarely, long-term survival, occasionally for $>20$ years, has been reported in $1-3 \%$ of metastatic breast cancer cases $(11,12)$. Several reports suggested that women with de novo disease exhibited better survival compared with patients with relapsed disease $(13,14)$, with the median survival of de novo stage IV disease ranging between 18 and 39 months. In our case, however, the patient only survived for 7 months after diagnosis of breast cancer. We consider the reasons for the patient's short survival to be the multiple metastatic sites, triple-negative phenotype and initial presentation with malignant pleural effusion and pericardial tamponade caused by pericardial effusion. The majority of deaths in metastatic breast cancer are not due to the primary tumor, but rather the result of metastasis to other organs (15). Visceral and central nervous system metastases are predictive of a poor prognosis and shorter survival compared with bone and soft tissue metastases $(8,16,17)$.

In the present case, the patient initially presented with malignant pleural and pericardial effusion, which were considered a grave prognostic sign. In previous studies, 


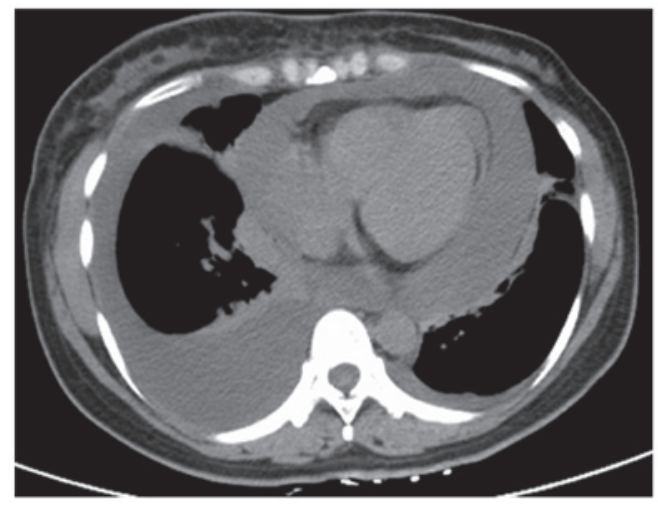

Figure 1. Plain chest computed tomography scan in October, 2013, revealing bilateral pleural effusions and a large amount of pericardial effusion with characteristics of tamponade.

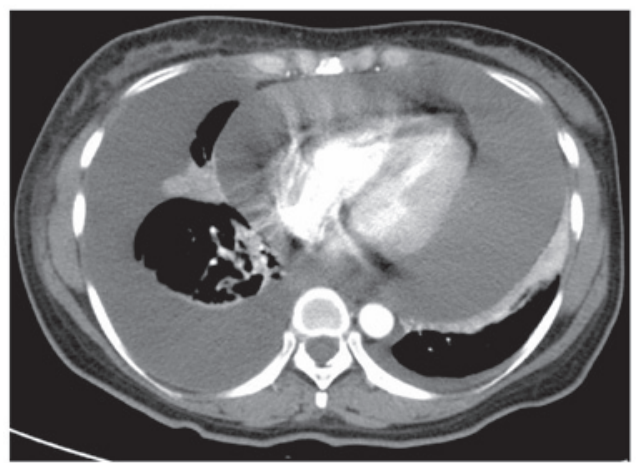

Figure 2. Enhanced chest computed tomography scan in February, 2014, showing a large amount of pericardial effusion and right pleural effusion.
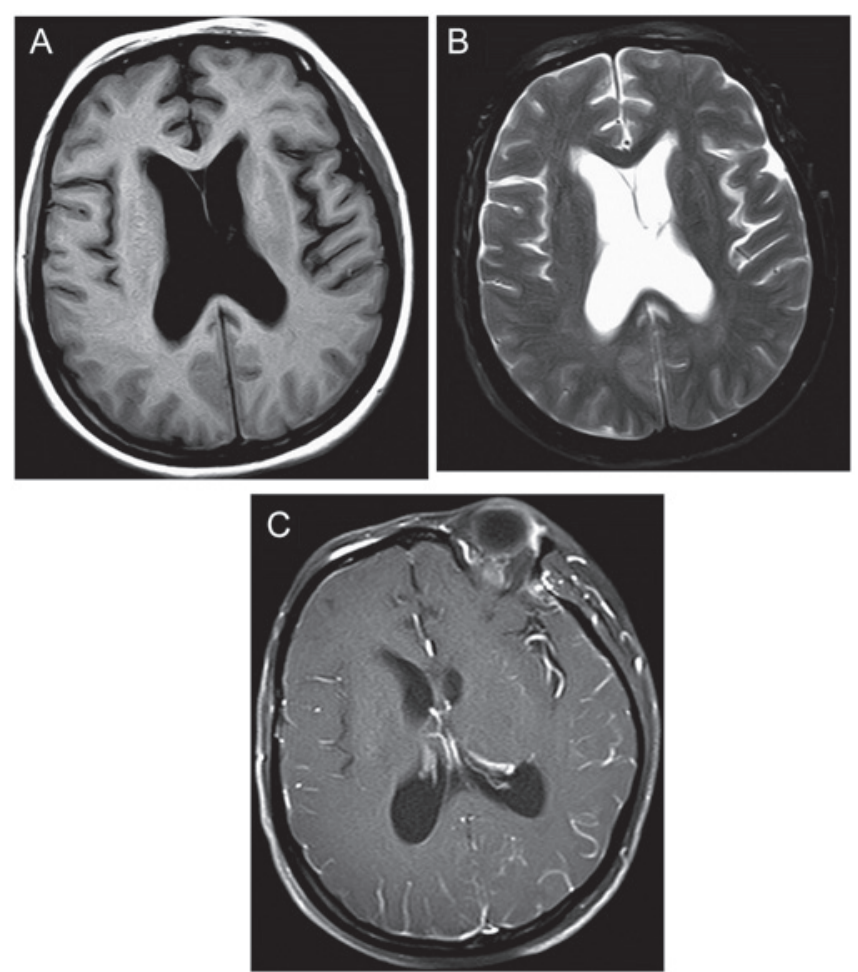

Figure 3. Magnetic resonance imaging head scan in March, 2014, showing narrowed bilateral frontal lobe grooves, hydrocephalus and enlarged ventricles, with mild enhancement of the gyrus in the enhanced scan. (A) T1-weighted plain scan. (B) T2-weighted scan. (C) T1-weighted enhanced scan.

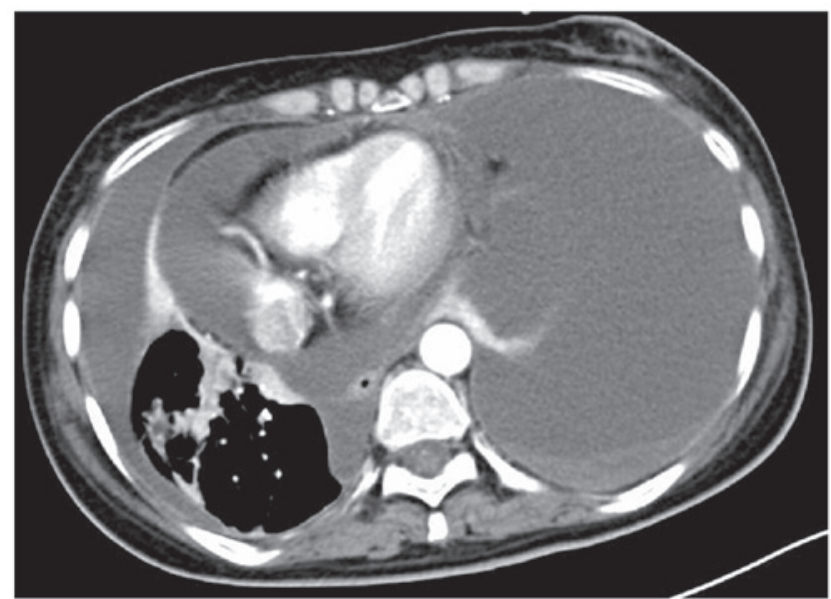

Figure 4. Enhanced chest computed tomography scan in April, 2014 showing pericardial effusion and bilateral pleural effusions.

$\sim 86 \%$ of patients succumbed within the first year and aproximately one-third within the first month. A median survival of 5-16 months has been reported in cases with malignant pleural effusion, and of 8-26 months in cases with malignant pericardial effusion (18-20). The prognosis of patients presenting with cardiac tamponade is dismal, with survival ranging from a few days to 14 months and a median survival of 5.5 months (21). Additionally, our patient developed meningeal metastases after 4.5 months. Prospective studies have reported a median overall survival of 9-30.3 weeks in patients with breast cancer following the diagnosis of meningeal metastases (22); in the present case, the patient survived for 2.5 months after the diagnosis of meningeal metastases. Finally, the prognosis of the triple-negative breast cancer patients is worse compared with that of other breast cancer phenotypes, as these tumors are more aggressive, with a higher incidence of distant metastases, particularly to visceral organs and the brain (23-25), and survival is shorter. Some studies demonstrated that the triple-negative breast cancer phenotype is an unfavorable characteristic in patients with malignant pleural effusion (26) and meningeal metastases (27), adversely affecting prognosis and reducing life expectancy $(17,24,26,28)$.

Breast cancer patients with malignant effusion and meningeal metastases are practically incurable; therefore, relieving the symptoms and improving the quality of life is very important. In our case, systemic chemotherapy was administered when the patient was in good overall condition, with thoracentesis and pericardiocentesis, as well as intracavitary instillation of cisplatin to control the malignant effusion. As regards meningeal metastases, WBRT was applied. However, although the disease was initially controlled, it progressed within a short time period, and the patient succumbed 7 months later.

In conclusion, metastasis to the pleura, pericardium and meninges, without metastases to other organs during the entire course of the disease, is a rare metastatic pattern of breast cancer. Treatment is usually aimed at relieving the symptoms and improving the quality of life, and the prognosis is typically poor. Clinicians should be aware of these rare metastatic patterns of breast cancer. 


\section{References}

1. Koo JS, Jung W and Jeong J: Metastatic breast cancer shows different immunohistochemical phenotype according to metastatic site. Tumori 96: 424-432, 2010.

2. Apffelstaedt JP, Van Zyl JA and Muller AG: Breast cancer complicated by pleural effusion: Patient characteristics and results of surgical management. J Surg Oncol 58: 173-175, 1995.

3. Evans TRJ, Stein RC, Pepper JR, Gazet JC, Ford HT and Coombes RC: A randomized prospective trial of surgical against medical tetracycline pleurodesis in the management of malignant pleural effusions secondary to breast-cancer. Eur J Cancer 29A: 316-319, 1993.

4. Posner MR, Cohen GI and Skarin AT: Pericardial disease in patients with cancer. The differentiation of malignant from idiopathic and radiation-induced pericarditis. Am J Med 71: 407-413, 1981.

5. Levitan Z, Kaplan AL and Gordon AN: Survival after malignant pericardial effusion and cardiac tamponade in advanced ovarian cancer. South Med J 83: 241-242, 1990.

6. Shetty J, Prasad K, Rao M and Nayak A: Isolated leptomeningeal metastases originating from infiltrating ductal carcinoma of breast - A case report. IJRRMS 2: 2012.

7. Jemal A, Siegel R, Ward E, Hao Y, Xu J, Murray T and Thun MJ: Cancer statistics, 2008. CA Cancer J Clin 58: 71-96, 2008.

8. Largillier R, Ferrero JM, Doyen J, Barriere J, Namer M, Mari V, Courdi A, Hannoun-Levi JM, Ettore F, Birtwisle-Peyrottes I, et al: Prognostic factors in 1,038 women with metastatic breast cancer. Ann Oncol 19: 2012-2019, 2008.

9. Bruna J, Simó M and Velasco R: Leptomeningeal metastases. Curr Treat Options Neurol 14: 402-415, 2012.

10. Ugenskiené R and Juozaityté E: The molecular mechanisms of breast cancer metastasis. Biologija 58: 2012.

11. Hortobagyi GN: Can we cure limited metastatic breast cancer? J Clin Oncol 20: 620-623, 2002.

12. Greenberg PA, Hortobagyi GN, Smith TL, Ziegler LD, Frye DK and Buzdar AU: Long-term follow-up of patients with complete remission following combination chemotherapy for metastatic breast cancer. J Clin Oncol 14: 2197-2205, 1996.

13. Dawood S, Broglio K, Ensor J, Hortobagyi GN and Giordano SH: Survival differences among women with de novo stage IV and relapsed breast cancer. Ann Oncol 21: 2169-2174, 2010.

14. Güth U, Magaton I, Huang DJ, Fisher R, Schötzau A and Vetter M: Primary and secondary distant metastatic breast cancer: Two sides of the same coin. Breast 23: 26-32, 2014.

15. Weigelt B, Peterse JL and van't Veer LJ: Breast cancer metastasis: Markers and models. Nat Rev Cancer 5: 591-602, 2005.
16. Solomayer EF, Diel IJ, Meyberg GC, Gollan C and Bastert G: Metastatic breast cancer: Clinical course, prognosis and therapy related to the first site of metastasis. Breast Cancer Res Treat 59: 271-278, 2000

17. Cutler SJ, Ardyce JA and Taylor SG III: Classification of patients with disseminated cancer of the breast. Cancer 24: 861-869, 1969.

18. Fentiman IS, Millis R, Sexton S and Hayward JL: Pleural effusion in breast cancer: A review of 105 cases. Cancer 47: 2087-2092, 1981.

19. Dieterich M, Goodman SN, Rojas-Corona RR, Emralino AB, Jimenez-Joseph D and Sherman ME: Multivariate analysis of prognostic features in malignant pleural effusions from breast cancer patients. Acta Cytol 38: 945-952, 1994.

20. Hirata T, Yonemori K, Hirakawa A, Shimizu C, Tamura K, Ando M, Katsumata N, Tanimoto M and Fujiwara Y: Efficacy of pleurodesis for malignant pleural effusions in breast cancer patients. Eur Respir J 38: 1425-1430, 2011.

21. Stitt VJ Jr: Breast carcinoma with pericardial metastasis. J Natl Med Assoc 79: 195-197, 1987.

22. Scott BJ and Kesari S: Leptomeningeal metastases in breast cancer. Am J Cancer Res 3: 117-126, 2013.

23. Rodríguez-Pinilla SM, Sarrió D, Honrado E, Hardisson D, Calero F, Benitez J and Palacios J: Prognostic significance of basal-like phenotype and fascin expression in node-negative invasive breast carcinomas. Clin Cancer Res 12: 1533-1539, 2006.

24. Chang BW, Decker RH, Haffty BG and Knisely JPS: Incidence of brain metastases in early-stage triple negative breast cancer patients. Int J Radiat Oncol Biol Phys 69 (Suppl): S210-S211, 2007.

25. Lin Y, Yin W, Yan T, Zhou L, Di G, Wu J, Shen Z, Shao Z and $\mathrm{Lu}$ J: Site-specific relapse pattern of the triple negative tumors in Chinese breast cancer patients. BMC Cancer 9: 342, 2009.

26. Lee SS, Ahn JH, Kim MK, Sym SJ, Gong G, Ahn SD, Kim SB and Kim WK: Brain metastases in breast cancer: Prognostic factors and management. Breast Cancer Res Treat 111: 523-530, 2008.

27. Santos GT, Prolla JC, Camillo ND, Zavalhia LS, Ranzi AD and Bica CG: Clinical and pathological factors influencing the survival of breast cancer patients with malignant pleural effusion. J Bras Pneumol 38: 487-493, 2012.

28. Chang EL and Lo S: Diagnosis and management of central nervous system metastases from breast cancer. Oncologist 8: 398-410, 2003. 